\title{
Psychological Adjustment to Craniofacial Conditions (Excluding Oral Clefts): A Review of the Literature
}

\begin{abstract}
Objective: A congenital craniofacial anomaly (CFA) is expected to impact upon several domains of psychological, emotional, and social functioning, yet no recent reviews have comprehensively summarised the available literature. Further, existing reviews tend to draw largely upon literature in the field of cleft lip and palate, and do not give substantive attention to other types of CFAs.
\end{abstract}

Design: A review of 41 papers published between January 2000 and March 2016 pertaining to psychological adjustment to CFAs.

Main Outcome Measures: Findings are presented according to four key domains of adjustment: Behaviour, Emotional Wellbeing, Social Experiences, and Appearance. In addition, treatment-related experiences, general psychological wellbeing and quality of life are explored.

Results: Current literature offers a contradictory picture of adjustment to a CFA throughout childhood and adulthood. Psychological adjustment appears to be comparable to norms and reference groups in approximately half of the included papers related to non-syndromic CFAs, while more variation is found across domains among samples with syndromic CFAs. Associations were found between psychological adjustment, physical health and cognitive function in several papers. The review identified a number of gaps in the literature, such as the inclusion of a wide range of diagnoses within research samples.

Conclusions: This review demonstrates the complexity of findings both within and across domains, and highlights a number of important methodological challenges. More research is needed to address these gaps in knowledge, and to identify ways of preventing distress and facilitating resilience in those born with CFAs.

Keywords: Craniofacial, behaviour, emotional, social, appearance, quality of life, treatment 


\section{Introduction}

A congenital craniofacial anomaly (CFA) is a broad term used to describe a wide range of diagnoses, which may be isolated to the head and neck or may form part of a wider genetic syndrome (Holmbeck \& Aspinall, 2015). Prevalence rates and characteristics vary widely across the many craniofacial conditions, affecting the form and function of the head (Buchanan, Xue, \& Hollier, 2014). Craniosynostosis results when one or more cranial structures fuse prematurely, and is relatively common, occurring in approximately one in every 2,000 live births (McCarthy, Warren, Bernstein, Burnett, Cunningham, Edmond et al., 2012). Treacher Collins syndrome is an autosomal dominant condition generally characterised by bilaterally symmetrical abnormalities of structures within the first and second branchial arches (Posnick \& Ruiz, 2000) and affects approximately one in 50,000 live births (Orphanet, 2015). Other conditions are much rarer, for example, Apert syndrome (approx. one in 65,000; Cohen, Kreiborg, Lammer, Cordero, Mastroiacovo, Erickson et al., 1992), Pfeiffer syndrome (approx. one in 100,000; Orphanet, 2015) and Crouzon syndrome (approx. one in 1,000,000; Orphanet, 2015); all syndromic forms of craniosynostosis. Depending on the condition and associated malformations, early surgical intervention may be necessary. Subject to the severity of the anomaly, multidisciplinary treatment may be required throughout childhood and into adulthood.

Although the majority of individuals affected by a CFA do not appear to encounter substantial psychological difficulties (Endriga \& Kapp-Simon, 1999), a significant proportion are thought to experience challenges in relation to behavioural (Speltz, Morton, Goodell, \& Clarren, 1993), social (Kapp-Simon \& McGuire, 1997) and emotional adjustment (Pillemer \& Cook, 1989). A high rate of learning disorders has also been identified (Kapp-Simon, 1998; Okkerse, Beemer, de Jong, Mellenbergh, Vaandrager, Vermeij-Keers, \& Heineman-de Boer, 2004). Ongoing treatment may represent a significant burden, in addition to the comments, questions, staring and teasing which can accompany a visible difference (Rumsey \& Harcourt, 2004).

Relative to research carried out among individuals born with one of the most common congenital craniofacial conditions, cleft lip and/or palate, investigations of psychological adjustment to other CFAs are scarce (Roberts \& Mathias, 2012). This is despite CFAs involving a more complex and varied level of routine aesthetic and functional surgical intervention, in addition to comparable anticipated effects on several domains of life. Given 
that the most recent comprehensive overview of the field was published by Endriga and Kapp-Simon in 1999, existing literature on CFAs needs an updated summary of the literature.

Review articles published more recently have focused on specific areas of functioning in relation to particular conditions, such as neurocognitive or neurobehavioural adjustment in individuals with craniosynostosis (Kapp-Simon, Speltz, Cunningham, Patel, \& Tomita, 2007; Knight, Anderson, Spencer-Smith, \& Da Costa, 2014; Speltz, Kapp-Simon, Cunningham, Marsh, \& Dawson, 2004), or surgical outcomes in a range of congenital and acquired conditions (Habal, 2013; Sarwer \& Crerand, 2002). While these reviews are helpful, it is also important to capture associations between the different domains of adjustment. It is possible for example, that an individual may be dissatisfied with their appearance, yet still report a good level of emotional adjustment (Feragen, Stock, \& Kvalem, 2015). In order to capture the complexity and variability of adjustment across the lifespan, and associations across different domains of adjustment, comprehensive reviews are needed.

More generally, articles which aim to review adjustment to CFAs predominantly refer to $\mathrm{CL} / \mathrm{P}$ literature, making it difficult to compare across diagnoses. Such observations also clearly highlight the lack of knowledge on adjustment to other congenital CFAs. Further, many of the existing review articles set out to provide an overview of the state of the field, rather than a systematic assessment of the literature. The aims of the present review were therefore as follows:

1) To summarise the literature published between January 2000 and March 2016 according to four key domains of psychological adjustment, in addition to treatmentrelated experiences and overall scores of psychological wellbeing and quality of life.

2) To investigate the impact of contributing variables and potential associations between the different domains of adjustment.

3) To present the methodological challenges apparent within the literature and to discuss implications for future research.

\section{Method}

\section{Inclusion Criteria}

All original, peer-reviewed articles pertaining to the psychological adjustment of individuals affected by CFA published between January 2000 and March 2016 were included. Papers using quantitative, qualitative and mixed methods were considered. No age restrictions of the 
participants were imposed. Articles relating to all types of syndromic and non-syndromic CFA were included. All methods of measurement, including self-reports, parent-reports and third-party reports (such as those obtained via clinicians or laypersons) were included.

\section{Exclusion Criteria}

Case studies and unpublished dissertations were excluded. Articles relating to "visible difference', 'disfigurement' or similar search terms were excluded where results pertaining to craniofacial conditions were not presented separately. Given a recent comprehensive literature review on psychological adjustment to CL/P (Stock \& Feragen, 2016), papers including individuals with this diagnosis were excluded, unless results were presented separately for other CFAs. Studies on 22q11.2. deletion syndrome (di George or velo-cardiofacial syndrome) were excluded, since the specificity of this condition and the high number of recent studies (Jonas, Montojo, \& Bearden, 2014; Philip \& Bassett, 2011; Swillen \& McDonald-McGinn, 2015) suggests a need for a review on this condition alone. In addition, articles focussing on cognitive function and development alone were excluded, since the literature on cognitive and neurobehavioural development in CFA is extensive and several reviews have been published on this domain during the last decade (Kapp-Simon et al., 2007; Knight et al., 2014; Speltz et al., 2004). Articles in which the primary aim was to trial the effects of medical/surgical intervention were also excluded. No literature reviews, systematic reviews, summary articles, book chapters or meta-analyses published during the search period were included, but were stored separately for reference.

Further details of included and excluded articles are provided in Figure 1.

\section{Search Strategy}

The PsycInfo, MEDLINE, and CINAHL databases were examined using search terms pertaining to central domains of psychological adjustment. Diagnostic search terms included: Craniofacial - Treacher Collins - Crouzon - Apert - Craniosynostos* - Microsomia - Muenke - Sæthre-Chotzen - Pfeiffer - Goldenhar - Trigonocephaly. Psychological search terms included: Adjustment - Behavio* - Psychol* - Emotion* - Social - Appearance - Satisfaction - Treatment - Quality of life - QOL. The Boolean operators OR were used between search terms within diagnostic terms and psychological search terms, while the operator AND was used between the two categories. Filters were used, restricting the searches to articles with abstracts available, papers written in English, involving humans and published since January 
2000. When connecting the diagnostic groups and the psychological terms, the search field was restricted to title and abstracts.

The reference lists of included articles and previous reviews were checked manually to reduce the likelihood of any abstracts being missed. The literature search was performed by the first author between January 2016 and March 2016. To assess quality control, the second author was given ten randomly selected abstracts to review. The evaluations made by the first and second authors were subsequently compared, and agreement was found to be high. Minor discrepancies were discussed until full agreement was reached.

\section{Domains of Adjustment}

In the context of this literature review, 'adjustment' was conceptualised as the process of the relative adaptation of an individual to the demands of their environmental context (see Seaton, 2009). The findings of each article were categorised according to four key domains of adjustment: Behaviour, Emotional Wellbeing, Social Experiences and Satisfaction with Appearance. Additionally, due to the extensive, multidisciplinary and long-term treatment involved in many CFAs, treatment-related issues was added as a separate category. Findings related to overall psychological wellbeing and/or quality of life (QoL) are also presented in a separate section. In the absence of conventionally agreed domains of adjustment, the development of each domain was guided by recent literature (e.g. Klassen, Tsangaris, Forrest, Wong, Pusic, Cano, Syed et al., 2012; Stock \& Feragen, 2016; Stock, Hammond, Owen, Kiff, Shanly, \& Rumsey, 2015). Decisions regarding the definition and content of each domain was discussed and reflected upon by the authors throughout the review process. Key findings were extracted and presented using the same terminology, definitions and interpretations used in the original articles. Similarly, the categorisation of a condition as syndromic or nonsyndromic was based on criteria used by the authors of the included papers.

Table 1 describes the categorisation of findings into these key domains.

\section{Results}

\section{Included Articles}

In total, 41 articles were included in this review. Of these articles, 39 were quantitative in nature, while only two studies used a qualitative approach. Most studies focussed on several domains of adjustment (see Table 2). An overview of each of the included articles is described in more detail in Table 3. 


\section{Summary of Findings across Key Domains of Adjustment}

Initially, the findings pertaining to each domain are described in relation to how individuals with CFA fare when compared to various comparison groups. Where appropriate, results pertaining to associations across different domains is presented. Finally, findings relating to overall psychological wellbeing or quality of life (QoL) are summarised.

\section{Behaviour}

\section{Behavioural conduct}

Among the 41 papers, 13 reported findings related to behavioural adjustment. The prevalence of behavioural problems (attention and hyperactivity disorders, oppositional defiant disorder, conduct disorder and features of the autistic spectrum disorder) in children with craniosynostoses was found to be similar to community-based samples when controlling for the co-occurrence of cognitive dysfunction (van der Vlugt, van der Meulen, Creemers, Verhulst, Hovius, \& Okkerse, 2012). Similarly, parents of children with hemifacial microsomia reported similar levels of behavioural problems as did matched controls (Dufton, Speltz, Kelly, Leroux, Collett, \& Werler, 2011). This was also the case for children with nonsyndromic craniosynostoses when comparing scores to siblings and norms (Boltshauser, Ludwig, Dietrich, \& Landolt, 2003) or matched controls (Cloonan, Collett, Speltz, Anderka, \& Werler, 2013). In contrast, behavioural problems were reported by $35 \%$ of parents of young people with non-syndromic craniosynostoses in another study, a statistically significant increase from the general population (Becker, Petersen, Kane, Cradock, Pilgtam, \& Marsh, 2005). According to an analysis of children with trigonocephaly (Kelleher, Murray, McGillivary, Kamel, Allcutt, \& Earley, 2006), 20\% of the children needed an assistant in the classroom due to behavioural challenges.

Among those with syndromic forms of craniosynostoses, elevated rates of behavioural problems were reported by parents when compared to norms (Maliepaard, Mathijssen, Oosterlaan, \& Okkerse, 2014). Another study (Bannink, Maliepaard, Raat, Joosten, \& Mathijssen, 2011) compared parent-reported behavioural problems between groups of children and young people with syndromic craniosynostoses and found that $50 \%$ of the young people with Muenke syndrome and 67\% of those with Apert syndrome had scores within the clinical range; figures that indicate statistically significant increases from the general population. Another study reported that less than $30 \%$ of young people with Apert syndrome had scores in the maladjustment range according to parent reports (Sarimski, 2001). Behavioural problems were more frequent among boys than girls (Bannink et al., 2011), and 
all young people with Muenke and Apert syndrome who had scores within the clinical range were boys (Bannink et al., 2011).

Two studies compared behavioural problems across subtypes of conditions. No differences were found between subtypes of non-syndromic trigonocephaly in relation to the frequency of behavioural problems (Kelleher et al., 2006), while some differences were found across subtypes of craniosynostoses (Becker et al., 2005).

Behavioural problems were associated with lower QoL, as well as physical symptoms, such as sleep apnoea (Bannink et al., 2011). Behavioural problems were also shown to be associated with underlying cognitive impairments in several studies (Maliepaard et al., 2014; van der Vlugt et al., 2012; van der Vlugt, van der Meulen, Creemers, Willemse, Lequin, \& Okkerse, 2009).

\section{Internalising and Externalising Behaviours}

Two studies on young children with single-suture craniosynostoses reported differences on externalising (Kapp-Simon, Collett, Barr-Schinzel, Cradock, Buono, Pietila, \& Speltz, 2012) and internalising behaviours (Kapp-Simon et al., 2012; Snyder \& Pope, 2010). However, overall differences were small and non-significant, except in one case of externalising behaviours, where $14.5 \%$ of the children with the condition had scores within the clinical range compared to $7.6 \%$ of the control group (Kapp-Simon et al., 2012). Another study reported a slight tendency for increased internalising symptoms in young people with sagittal craniosynostoses (Boltshauser et al., 2003). No differences between subtypes of craniosynostoses were found (Kapp-Simon et al., 2012). A study on children with hemifacial macrosomia reported no differences between parent ratings and norms (Snyder \& Pope, 2010), while teachers reported a higher frequency of internalising behaviours than parents compared to matched controls (Dufton et al., 2011; Werler, Starr, Cloonan, \& Speltz, 2009).

In contrast, syndromic CFAs were more highly associated with scores within the clinical range. One study found that $32 \%(n=8 / 25)$ of young people with Apert syndrome showed problems with internalising or externalising behaviours (Sarimski, 2001). Another study that included children with a wide range of different syndromic craniosynostoses (Maliepaard et al., 2014) also reported higher rates of problems with internalising behaviours compared to norms. Problems with internalising behaviours were shown to be linked to impaired cognitive function in two studies (Sarimski, 2001; van der Vlugt et al., 2009), and one study explicitly 
showed that the lower the intelligence quotient, the higher the prevalence of behavioural problems (van der Vlugt et al., 2009).

\section{Emotional Wellbeing}

\section{Emotional Functioning}

Several studies used quality of life or general adjustment measures which include subscales of emotional well-being. Among the 41 included articles, 17 reported findings in relation to emotional adjustment specifically.

According to parents, young children with non-syndromic craniosynostoses had 'normal' emotional development when compared to a control group (Knudsen, Maltese, Tarnow, Tovetjarn, \& Kolby, 2012). Another study on young people with sagittal craniosynostoses (Boltshauser et al., 2003) revealed lower scores in regard to positive emotions, again rated by parents. However, scores for negative emotions were not significantly different when compared to data collected from siblings and/or norm data (Boltshauser et al., 2003).

Parents of children with complex craniosynostoses reported no significant differences in relation to emotional functioning, although mean scores for the whole sample were indicative of more emotional problems than norms (Maliepaard et al., 2014). One study found an impact on the emotional wellbeing of young people with syndromic craniosynostoses, as rated by parents, particularly in cases of Apert syndrome and complex craniosynostoses, as well as an impact on parents' own emotional wellbeing (Bannink, Maliepaard, Raat, Joosten, \& Mathijssen, 2010). Parents of young people with Apert syndrome also reported occasional incidences of depressed mood in $32 \%$ of the children (Sarimski, 2001). In a study by Tovetjärn, Tarnow, Maltese, Fischer, Sahlin, \& Kolby (2012), 73.9\% of adults with Apert syndrome reported periods of depressive mood, compared to $23.3 \%$ in matched controls.

For parents of young people affected by Crouzon and Pfeiffer syndrome, emotional wellbeing scores were close to normal (Bannink et al., 2010). Similarly, when asking parents to rate their child's emotional adjustment, Maliepaard et al. (2014) did not find any differences in relation to emotional functioning among children with Crouzon, Pfeiffer or Sæthre-Chotzen syndrome, despite mean scores for the whole sample suggesting more emotional problems than norms (Maliepaard et al., 2014). Adults with syndromic craniofacial conditions reported mean scores of depression and anxiety within the normal range and comparable to norms in one study (Roberts \& Mathias, 2012). In contrast, 33.3\% of young adults with Crouzon syndrome reported periods of depressive mood compared to $19.1 \%$ in a matched control 
group. Young adults with Crouzon syndrome also reported having suicidal thoughts at some time (Fischer, Tovetjärn, Maltese, Sahlin, Tarnow, \& Kolby, 2014). However, there were wide variations within the group, pointing to important individual differences (Fischer et al., 2014). In another study, adults with Treacher Collins syndrome reported less problems with anxiety, but more problems with depression than those with cherubism (Geirdal, Saltnes, Storhaug, Åsten, Nordgarden, \& Jensen, 2015).

Associations between physical symptoms such as sleep apnoea and emotional problems were not found in young people with syndromic craniosynostoses (Bannink et al., 2011). Further, having or not having completed surgical treatment did not affect mean scores of emotional functioning (Roberts \& Mathias, 2013). Emotional problems had no or a limited influence on measures of health-related quality of life (HRQoL) in young people with syndromic and complex craniosynostoses (de Jong, Maliepaard, Bannink, Raat, \& Mathijssen, 2012). In contrast, emotional functioning has been demonstrated to be associated with cognitive function. For example, children with IQ scores of $<85$ had a two to three times higher risk of having emotional problems than children with intelligence scores within the normal range (Maliepaard et al., 2014).

Several studies reported associations between emotional adjustment and social experiences. Higher rates of perceived discrimination were found to be associated with higher levels of depression and anxiety in young adults with syndromic craniofacial conditions (Roberts, 2014). A similar association was reported in Roberts and Mathias (2012), where emotional problems were related to limitations in social activities. Further, Roberts and Mathias (2013) found that anxiety was predicted by social support, self-esteem and fear of negative evaluation, while depression was predicted by lower levels of perceived social support and poorer self-esteem.

\section{Self-Concept and Self-Esteem}

In a qualitative study of adolescents with Treacher Collins syndrome (Beaune, Forrest, \& Keith, 2004), young people described how they had dealt with interactions that were emotionally demanding or uncomfortable by moving from negatively-based response strategies to more positive and assertive strategies (Beaune et al., 2004). These adolescents felt they had reconstructed the meanings of the difference they had to live with, and as a result experienced an increase in self-acceptance (Beaune et al., 2004). Further, the adolescents also shared how excelling in other areas of life had become a coping strategy which minimised 
their perceived difference and highlighted their competencies, thus helping them to develop a successful pathway (Beaune et al., 2004). In a qualitative study of adults with Crouzon syndrome, similar findings were reported (Stavropoulos, Hallberg, Mohlin, \& Hagberg, 2011); for example, participants stated that utilising positive coping strategies helped to protect their self-image. Those who constructed positive identities were those who conceptualised their difference not as a personal failure, but as a situation that could be challenged and resisted (Stavropoulos et al., 2011). In a quantitative study (Roberts, 2014), adults with syndromic craniofacial conditions reported higher self-esteem than a community sample. Similarly, a study on adolescents and adults with Apert syndrome presented selfreported positive feelings, self-esteem and positive body image (Raposo-Amaral, RaposoAmaral, Garcia Neto, Farias, \& Somensi, 2012).

An association between perceived discrimination and lower self-esteem was found in a sample of adults with syndromic craniofacial conditions (Roberts, 2014). Associations were also found between self-esteem and emotional problems, such as depression or anxiety (Roberts \& Mathias, 2013).

\section{Social Experiences}

\section{Social Functioning}

Almost half of the included studies investigated social functioning and social experiences (16/41 articles). Parents of children, adolescents and young adults with sagittal craniosynostoses reported scores on social functioning to be within the normal range (Boltshauser et al., 2003). The authors concluded that the participating individuals had developed coping strategies allowing them to function in a competent way, both psychologically and socially (Boltshauser et al., 2003). Other studies have also found children with non-syndromic craniosynostoses (Cloonan et al., 2013) and hemifacial microsomia (Dufton et al., 2011) to have levels of social competence similar to matched control groups. Another study revealed lower social functioning in this diagnostic group compared to unaffected peers, also based on parent reports (Khetani, Collett, Speltz, \& Werler, 2013). Social problems were also identified in young children with single-suture craniosynostoses (Kapp-Simon et al., 2012), although low effect sizes were reported. In a study by Dufton and colleagues (2011), teachers reported lower social competence and peer acceptance in children with hemifacial microsomia compared to matched controls. However, few of the children received scores above the clinical range. 
In the case of Crouzon syndrome, affected adults described how they actively launched themselves into social situations, with attempts to be charismatic, open, sociable, humorous and focussed upon others, in order to help other people to see beyond their facial appearance (Stavropoulos et al., 2011). In contrast, and according to parent reports, 56\% of children and adolescents with Apert syndrome reported clinically significant social problems (Sarimski, 2001). Similarly, adults with syndromic craniosynostoses seemed to struggle more than norms in relation to social problems and social functioning (Roberts \& Mathias, 2012). In young people and adults with Treacher Collins syndrome, the presence of sleep apnoea impacted significantly on social functioning (Geirdal, Øverland, Heimdal, Storhaug, Åsten, \& Akre, 2013).

\section{Social Reactions to a Craniofacial Difference}

Adolescents with Treacher Collins syndrome described frequent overt as well as subtle social reactions from others, which they found uncomfortable, such as staring or whispering (Beaune et al., 2004). They also shared how social reactions to their difference created an internal struggle between feeling the same as others while also being different (Beaune et al., 2004). Perceived daily discrimination was reported by a minority of adults with syndromic craniofacial conditions (Roberts, 2014). In spite of potentially negative social reactions, fear of negative evaluations was found to be comparable to norms in a group of adults with syndromic craniofacial conditions (Roberts and Mathias, 2012). In a sample of children and adolescents with Apert syndrome, one third reacted by socially withdrawing, according to their parents (Sarimski, 2001). Adults with Crouzon syndrome offered the perspective that avoiding situations where they felt exposed could occasionally be a helpful coping strategy (Stavropoulos et al., 2011). Nonetheless, fear of negative evaluation predicted levels of general anxiety in adults with syndromic CFAs (Roberts \& Mathias, 2013).

\section{Teasing}

Only two studies specifically explored the prevalence of teasing experienced by individuals with CFAs. A total of $40 \%$ of children, adolescents and young adults with sagittal craniosynostoses reported experiences of teasing (Boltshauser et al., 2003), while $65.5 \%$ of adults with Crouzon syndrome reported being teased at some time, compared to $20.1 \%$ in a matched control group (Fischer et al., 2014).

The adolescents participating in the study by Beaune and colleagues (2004) reported that positive coping strategies had helped them to cope with any teasing. Positive coping strategies 
that counterbalanced experiences of stigmatisation and teasing were also described in young people and adults with Apert syndrome (Raposo-Amaral et al., 2012).

\section{Social Acceptance and Support}

Adolescents with Treacher Collins syndrome described how forming friendships and 'fitting in' became a protective factor in their lives (Beaune et al., 2004). Nevertheless, adults with syndromic craniosynostoses reported lower levels of social support from others and from peers than norms (Roberts \& Mathias, 2012). Similarly, adults with Apert syndrome (Tovetjärn et al., 2012) reported fewer friends than matched controls. Social support from family, on the other hand, was found to be within the normal range in adults with CFA (Roberts \& Mathias, 2012). Females reported higher levels of social support than males, as has been demonstrated within the general population (Roberts \& Mathias, 2012).

Associations between emotional and social adjustment were found in several studies. As an example, perceived social support and self-esteem explained $44 \%$ of the variance in depressive symptoms in adults with craniofacial conditions (Roberts \& Mathias, 2013). Levels of social support were also found to impact on self-perceived physical QoL (Roberts \& Mathias, 2013), while no associations were found between physical symptoms and psychosocial adjustment in another study (Warschausky, Kay, Buchman, Halberg, \& Berger, 2002). Social support was also found to partially mediate the relationship between social discrimination and psychological health (Roberts, 2014). Further, commitment to social activities was found to protect adults with Crouzon syndrome in relation to feeling selfconscious about their appearance, which also increased well-being (Stavropoulos et al., 2011). Social acceptance and subjective experiences of discrimination were not related to the type of condition or to socioeconomic status (Roberts, 2014).

\section{Romantic Relationships}

Parents of male adolescents with sagittal craniosynostoses reported their children to have similar romantic expectations as their peers (Boltshauser et al., 2003). Similarly, most young adolescents with Treacher Collins reported positive romantic experiences and had positive expectations of future romantic relationships (Beaune et al., 2004). In contrast, a sample of adults with Crouzon syndrome felt 'locked out' of this part of life, and described how they wanted to find a life partner who had also experienced similar life challenges (Stavropoulos et al., 2011). The more 'normal' adults felt, the more they believed in their chances of finding a romantic partner (Stavropoulos et al., 2011). In two other studies, adults with Apert 
(Tovetjarn et al., 2012) and Crouzon syndrome (Fischer et al., 2014) reported fewer romantic and sexual experiences than matched controls. Two studies on adults with syndromic craniofacial conditions indicated that they were less likely to marry and to have children when compared to norms (Fischer et al., 2014; Roberts \& Mathias, 2012).

\section{Satisfaction with Appearance}

Parent and Patient Satisfaction with Appearance

More than a quarter of the studies investigated appearance-related issues (13/41 articles). Adolescents with Treacher Collins syndrome (Beaune et al., 2004) described the psychological challenge of living with an unusual appearance and how their self-perceptions contained feelings of 'sameness' and 'difference'. In another study on adults with Treacher Collins, participants were least satisfied with their ears, facial profile, and eyelids (Plomp, Versnel, Lieshout, Poublon, \& Mathijssen, 2013), while being relatively satisfied with nasal appearance (Plomp et al., 2013; Plomp, Mathijssen, Moolenburgh, van Montfort, van der Meulen, \& Poublon, 2015), but less so than the control group. Findings of relative satisfaction was confirmed among adults with a wide variation of syndromic craniofacial conditions (Roberts \& Mathias, 2012), and among parents and children treated for trigonocephaly (Kelleher, Murray, McGillivary, Kamel, Allcutt, \& Earley, 2007), and parents of children with sagittal craniosynostoses (Boltshauser et al., 2003). In spite of mean scores suggesting adult patients to have more appearance concerns than norms, scores were within the high average range for both groups, indicating positive satisfaction with appearance (Roberts \& Mathias, 2012, 2013). Young people and adults with Apert syndrome also described positive feelings about their appearance (Raposo-Amaral et al., 2012).

Associations with other domains of adjustment were reported. Perceived discrimination, for example, was found to be related to lower ratings of subjective appearance in adults with syndromic craniofacial conditions (Roberts, 2014). Satisfaction with appearance was not associated with gender, age, or level of education in adults with syndromic craniofacial conditions (Roberts \& Mathias, 2013), while mothers' perceptions of the visibility of their infant's craniosynostosis was associated with higher levels of parental stress (Rosenberg, Kapp-Simon, Starr, Cradock, \& Speltz, 2011).

\section{Perceptions of Appearance among Laypersons}

A longitudinal cohort study of young children with single-suture craniosynostoses investigated laypersons' perceptions of attractiveness and appearance from infancy to 36 
months (Collett, Gray, Kapp-Simon, Birgfeld, Cunningham, Rudo-Stern et al., 2013). Results suggested that laypersons gave lower appearance ratings to children with craniosynostoses than controls at each time point. Over time, laypersons' appearance ratings decreased for both the patient and control groups (Collett et al., 2013). Differences between subtypes of craniosynostoses were found, and earlier surgery predicted better appearance outcomes, according to laypersons' ratings (Collett et al., 2013). Another study, based on photographs of children and young people with cerebrocraniofacial dysplasia with or without brain malformations, revealed differences in appearance and impairment ratings across subtypes of the condition, as well as across the gender of ratees and raters (Okkerse, Beemer, Cordia-de Haan, Heineman-de Boer, Mellenbergh, \& Wolters, 2001). Girls were given lower appearance ratings and higher ratings on malformation impairment than boys, and female raters assigned higher appearance ratings and lower impairment ratings than did male raters (Okkerse et al., 2001).

\section{Treatment-Related Experiences}

\section{Satisfaction with Treatment}

A quarter of the studies explored treatment-related issues (11/41 articles). Mothers of children with craniosynostoses were pleased with their child's appearance after surgery (WongGibbons, Kancherla, Romitti, Tyler, Damiano, Druschel et al., 2009). Similarly, a prospective study on young children with metopic suture synostoses (Kunz, Lehner, Heger, Armbruster, Weigand, Mast, \& Peraud, 2014) indicated that $92 \%$ of the parents were highly satisfied with their child's appearance after surgery. However, a discrepancy was found between the surgeon's perception of an optimal appearance outcome, and parents' satisfaction with the results of surgery in some cases. Last, parents and adult patients with non-syndromic trigonocephaly (Kelleher et al., 2007) rated satisfaction with cosmetic outcomes of surgery and scar appearance as excellent in $40-50 \%$ of the cases, while only $4 \%$ rated outcomes as fair. No correlations were found between the changes that occurred in head circumference and the degree of satisfaction as expressed by the patients or their families (Kelleher et al., 2007).

Few studies specifically measured satisfaction with aspects of treatment. Parents of young children with craniosynostoses mostly reported satisfaction with condition-related information and support from health professionals. Concordantly, access to treatment and care was rated as satisfactory (Wong-Gibbons et al., 2009). In another study, parents of young children also with craniosynostoses, reported an association between satisfaction with treatment and speech problems (Knudsen et al., 2012). Adolescents with Treacher Collins actively recommended 
healthcare professionals to be communicative, to listen, to show kindness and compassion, to treat patients like people and not as a medical condition, to have similar expectations of them as they would their unaffected peers, to provide information about the condition and to prepare them for future treatment (Beaune et al., 2004). In another study, adults with Treacher Collins had high levels of desire for future treatment, predominantly related to eyelid reconstruction (Plomp et al., 2013).

\section{Treatment Decisions}

One study investigated parents' attitudes towards surgical treatment in cases of craniosynostoses (Ozgur, Aryan, Ibrahim, Soliman, Meltzer, Cohen, \& Levy, 2006). The study contacted parents who had initially chosen not to have their children operated, in order to investigate their initial attitudes towards surgical treatment. The dominant view against early surgery was the idea that the potential results weren't worth the risks. Later on, the cumulative effects of childhood and schoolyard teasing proved to overpower the parents' initial feelings regarding their perceived risks of surgery (Ozgur et al., 2006).

Only one included study investigated patients' perception of treatment decisions. In this study, adults with Crouzon syndrome described psychological challenges in relation to deciding when to stop treatment (Stavropoulos et al., 2011).

\section{Psychological Adjustment to Treatment}

Adults with Crouzon syndrome (Stavropoulos et al., 2011) talked about their wishes for surgical treatment during the adolescent years in particular. Nevertheless, the same adults described how surgical treatment had been physically and psychologically demanding (Stavropoulos et al., 2011). A changed appearance following surgery could trigger identity crises, but also had the potential to enhance well-being (Stavropoulos et al., 2011).

\section{General Adjustment and Wellbeing}

Quality of Life

More than half of the papers investigated QoL or HRQoL and/or general adjustment (22/41 articles). Parents of children with non-syndromic craniosynostoses (Cloonan et al., 2013) and hemifacial microsomia (Khetani et al., 2013) reported lower HRQoL compared to matched controls, and compared to young people with an oral cleft (Warschausky et al., 2002). In another study (Geels, Kieffer, Hoogstraten, \& Prahl-Andersen, 2008), parent and self-reports indicated good oral HRQoL in children with a wide range of syndromic and non-syndromic craniofacial conditions. Similarly, parents of infants with single-suture craniosynostosis 
reported similar levels of general stress as did the control group (Rosenberg et al., 2011), and QOL measures within the normal range (Boltshauser et al., 2003). Interestingly, fathers were more likely to perceive their child to be more stressed than were mothers, while mothers reported higher levels of parental stress than did fathers (Rosenberg et al., 2011).

Parents of children and adolescents with syndromic craniosynostoses had significantly lower scores on QoL measures than norms, especially for those parents of children with Apert, Crouzon and Pfeiffer syndromes (Bannink et al., 2010; de Jong et al., 2012), as well as complex craniosynostoses (Bannink et al., 2011). Parents of children with Apert syndrome reported poorer physical functioning compared to norms, and a major impact of the diagnosis on the child's psychosocial functioning (Bannink et al., 2010).

In contrast, adults with a wide range of syndromic craniofacial conditions reported consistently positive perceptions of QoL comparable to norms (Prescott, Redfors, Rustad, Eiklid, Geirdal, Storhaug, \& Jensen, 2013; Roberts \& Mathias, 2012). More specifically, adults with Crouzon syndrome had similar estimations of their somatic health as matched controls, and an equal general positive attitude to life (Fischer et al., 2014), as was found in adults with Apert syndrome (Tovetjärn et al., 2012). Another study found that participants with Treacher Collins syndrome reported lower levels of overall QoL, well-being and mental health than patients with cherubism (Geirdal et al., 2015). In another study, patients with cherubism reported similar levels of QoL as a large reference group (Prescott et al., 2013). A comparison of children and adults with Crouzon and Apert syndrome confirmed satisfactory QoL across both conditions (Raposo-Amaral et al., 2012; Raposo-Amaral, Neto, Denadai, Raposo-Amaral, \& Raposo-Amaral, 2014).

Overall, parents tended to report more child-related problems than as indicated by self-reports (Bannink et al., 2010; Khetani et al., 2013). The same tendency was found in another study comparing self- and parent reports; however, differences were small and non-significant (Geels et al., 2008).

Associations between physical well-being and elements of QoL were reported by several studies of syndromic craniosynostoses, including associations with sleep apnoea and/or other medical problems (Bannink et al., 2010, 2011; Geirdal et al., 2013; Tovetjärn et al., 2012), difficulties with vision or speech (de Jong et al., 2012), parental emotional status (Warschausky et al., 2002) and measures of cognitive function (de Jong et al., 2012; RaposoAmaral et al., 2014). 
There were no associations between QoL and age (Geirdal et al., 2013; Warschausky et al., 2002) or gender (Roberts \& Mathias, 2013; Warschausky et al., 2002). On the other hand, self-perceived negative discrimination (Roberts, 2014) and emotional adjustment (Roberts \& Mathias, 2013), were associated with reduced satisfaction with life. Similarly, mothers of infants with single-suture craniosynostoses reported that the more visible they felt the condition was to other people, the higher levels of stress they experienced (Rosenberg et al., 2011).

\section{General Adjustment}

Only two studies explicitly reported total adjustment scores. Overall psychological adjustment was well within the normal range for children and adults with sagittal craniosynostoses, according to parent reports and when compared to siblings and norms (Boltshauser et al., 2003). Another study on adolescents with Apert syndrome found that $32 \%$ had total adjustment scores within the clinical range (Sarimski, 2001).

Associations were reported in three studies. One study identified an association between positive adjustment and active coping strategies, social support seeking, and use of avoidance strategies (Roberts \& Shute, 2012). When controlling for baseline levels of adjustment, however, associations with coping strategies became non-significant (Roberts \& Shute, 2012). Associations were also demonstrated between overall adjustment scores and health concerns (Warschausky et al., 2002) and with intellectual functioning according to parent reports (Maliepaard et al., 2014). No effects of age were found in the adjustment of adolescents with syndromic craniofacial differences (Roberts \& Shute, 2012).

\section{Discussion}

\section{Synthesis of Findings}

This review examined literature pertaining to psychological adjustment to a congenital craniofacial condition published between January 2000 and March 2016. Findings were categorised according to four key domains: 'Behaviour', 'Emotional Wellbeing', 'Social Experiences' and 'Satisfaction with Appearance', in addition to parents' and patients' treatment-related experiences, general psychological wellbeing and QoL. Associations between the different domains of adjustment were also examined.

The most recent comprehensive overview of the field was published by Endriga and KappSimon in 1999. The authors concluded that while the majority of children with CFAs demonstrate psychological functioning within the normal range, subgroups of children appear 
to be at risk in relation to a number of domains. The present review examined whether recent literature can further explain variations in psychological adjustment among children, adolescents and adults with congenital CFAs.

The frequency of behavioural problems seemed to be within the normal range in the majority of papers based on samples of non-syndromic conditions, while all of the studies focusing on syndromic conditions reported more behavioural challenges compared to control groups. Several studies demonstrated a close association between behavioural adjustment and cognitive impairments, suggesting that studies should consider controlling for cognitive function to avoid bias in the results. Another association reported by two studies was the link between behavioural problems and physical and/or medical concerns. This finding could help to explain the higher prevalence of behavioural problems among individuals with conditions that are associated with more physical and medical concerns than others, such as Apert and Muenke syndrome (Bannink et al., 2011). The current literature therefore points to the need for routine screening of important underlying background factors, such as cognitive development and physical health, so that the investigation of other psychological domains can be corrected for impaired neurological functioning. The assessment of cognitive function would also provide an important basis for school arrangements, in order to optimise learning strategies (Broder, 2001).

Within this review, two studies reported emotional adjustment in non-syndromic craniosynostoses to be comparable to reference groups. In comparison, a syndromic CFA, combined with cognitive impairments (e.g. as in Apert syndrome), may impact more considerably on the emotional wellbeing of both the individual and their parents. In the case of other CFAs, such as Crouzon syndrome and Treacher Collins syndrome, findings are less clear, and wide variations within and across groups point to important individual differences. Several studies indicated a high level of self-esteem and the use of positive coping strategies among adults with Treacher Collins syndrome and Crouzon syndrome. The findings of this review therefore suggest that investigating positive, as well as negative impacts of CFAs could provide a better understanding of the complexity of adjustment, and useful insight for promoting resilience within clinical practice (Feragen, 2012). Associations between emotional and social adjustment also suggest that interventions which can foster improvements in one domain could result in improvements in associated domains (Feragen et al., 2015). 
Overall, social functioning among those with non-syndromic CFAs was similar to comparison groups or norms in approximately half of the included studies. In contrast, almost all studies pertaining to syndromic cases reported social challenges, suggesting that the presence of a syndrome may negatively impact upon social experiences. Another important aspect of living with a visible difference such as a CFA, is repeated exposure to the social reactions of others, such as staring, comments, questions, or teasing (Rumsey \& Harcourt, 2004). Two studies suggested that the frequency of teasing was higher in children and adults with CFAs compared to control groups. Nonetheless, qualitative accounts of adults with Crouzon and Treacher Collins syndrome illustrated how some individuals are able to develop positive coping strategies and resilience to address these social challenges. Such strategies have useful implications for clinical practice; for example, staring could be interpreted as curiosity, rather than a threat (Partridge, 2010), thereby reducing the emotional impact of the event. Indeed, the associations identified in this review between social and emotional adjustment indicate the need to help individuals with CFAs manage the potential impact of their visible difference in social situations, and to strengthen social skills in order to prevent the development of emotional difficulties. Cognitive-behavioural techniques (see Clarke, Thompson, Jenkinson, Rumsey, \& Newell, 2013) have shown promise in this area. In addition, the literature included in this review indicated that positive social experiences and perceived social support may act as a buffer and protect individuals against negative experiences. Nonetheless, there remains a need for further rigorous research using appropriate outcome measures, in order to evaluate the effectiveness of such interventions (Jenkinson, Williamson, Byron-Daniel, \& Moss, 2015; Norman \& Moss, 2007).

Findings related to romantic experiences and relationships were largely based on samples with syndromic conditions, and revealed significant difficulties among many young people and adults with CFAs. Studies from the fields of visible difference (Griffiths, Williamson, \& Rumsey, 2012) and oral clefts (Feragen, Stock, Sharratt, \& Kvalem, 2016) have suggested that while a visible condition poses several challenges for those affected, the overall impact on adjustment may be low. Nonetheless, the lack of research on the romantic experiences among young people and adults with CFA stands in sharp contrast to the importance of this variable in many people's lives (Furman \& Shaffer, 2003). Further exploration of the impact of syndromic and non-syndromic CFAs on romantic experiences and relationships is therefore needed. 
When reported by parents, satisfaction with appearance was found to be high. However, it is possible that parents may provide positive reports of their child's appearance in any case. Importantly, parent perceptions of treatment outcome did not often correlate with surgeons' ratings, pointing to a well-known discrepancy between subjective and more "objective" ratings of appearance within the visible difference literature (Moss, 2005). Results from studies of laypersons' perceptions revealed a tendency to assign lower appearance ratings to individuals with CFAs, particularly for girls. Negative attitudes among members of the general public toward individuals with a visible difference have been demonstrated within the wider literature (Grandfield, Thompson, \& Turpin, 2005; Partridge \& Julian, 2008; Stock, Whale, Jenkinson, Rumsey, \& Fox, 2013), illustrating the challenges of adjusting to a visible difference in a society with increasingly high appearance standards and values (see Grogan, 2008). As important example highlighted in this review is the description of balancing feelings of 'sameness' and 'difference' when trying to 'fit in' with peers. In spite of this, subjective satisfaction with appearance was reportedly good overall among adolescents and adults with a wide variety of syndromic CFAs. Current findings pertaining to appearancerelated adjustment in individuals with CFA are therefore difficult to interpret and require further investigation. In addition, the diversity in findings within this domain of adjustment calls for researchers to move away from the paradigm that an observable visible difference automatically leads to dissatisfaction with appearance, and toward an exploration of the factors which may impact upon an individual's self-perceptions. Further investigation of the relationship between appearance and other domains of adjustment could also shed light on the processes involved in the development of appearance dissatisfaction, and guide future research in this area.

Unexpectedly, given the complexity and long-term treatment pathway for many CFAs, few studies specifically investigated treatment-related experiences. One study described adolescence as being a time of strong wishes for surgical treatment. However, participants also described a tension between post-surgical feelings of enhanced well-being, and crises triggered by changes in their appearance. Young adults with Treacher Collins syndrome highlighted the importance of information and listening skills, providing important 'guidelines' for health professionals. Another study investigated the attitudes of parents who had initially chosen not to have their children operated, but who subsequently re-evaluated this decision as a result of the child's perceptions of negative social experiences. Such studies illustrate the need for a balanced discussion with patients and/or their parents in relation to 
treatment opportunities, encompassing aesthetic, developmental and psychosocial aspects. When looking at this area as a whole, and given the centrality of extensive and long-term treatment for many CFAs, the emotional and psychological impact of treatment on patients and their families is a key subject for future research.

When measured in children with non-syndromic CFAs, QoL was found to be lower in one study, similar to norms in another, and high in yet another study. Conflicting findings were also found in samples with syndromic CFAs, where approximately half of the included studies reported lower scores in relation to QoL when compared to norms or to other medical conditions. These discrepancies could be partially explained by associations between QoL and a higher level of developmental and cognitive problems in those with syndromic forms of CFAs. Nonetheless, adults with a wide range of syndromic CFAs reported consistently higher or similar perceptions of QoL compared to norms in several studies. While only two studies investigated 'general adjustment', scores were reported to be within the normal range among non-syndromic cases, and poorer in cases of syndromic CFA. Such findings illustrate the complexity of the construct of 'QoL', and the multifaceted nature of adjustment (see Nelson, Glenny, Kirk, \& Caress, 2012; Rumsey \& Stock, 2013). Additionally, the impact of a given factor may vary across different domains of adjustment. For example, results from the present review seem to point to an association between cognitive impairments found in many syndromic CFAs, and behavioural or social problems, while QoL or satisfaction with appearance seemed less affected by potential cognitive difficulties. Thus, investigation of the various psychological domains may be more useful than relying on a single summary score alone, in order to more clearly identify where areas of difficulty and strength may lie. In addition, there is a clear need to further investigate potential differences between the various CFA diagnoses, in order to identify those children who are most vulnerable to psychological distress. As well as indicating a high degree of individual and condition-specific variation, inconsistencies in findings within the literature are likely also due to methodological disparity.

\section{Methodological Considerations}

The present review has highlighted a number of methodological challenges within the field of psychological adjustment to CFAs. First, and as anticipated, relatively few studies drew upon large samples. Half of the included studies had less than 50 participants, seven studies had between 50 and 100 participants, and the remaining thirteen studies had more than 100 participants (two of which were based on photographs). Similarly, participants presenting 
with a variety of different conditions and representing a broad age range were often mixed within one sample. This is understandable when conditions are rare, yet this approach renders analysis of relevant subgroups and potentially confounding factors extremely difficult (Roberts \& Mathias, 2012; Knight et al., 2014). Further, there are not only differences apparent between conditions, but also within conditions (for example, sagittal, complex or single-suture craniosynostosis, and syndromic versus non-syndromic cases). In addition, a large variation in the timing of studies relative to the treatment journey was observed; for example, the combination of treated and untreated case series within a single sample. Multicentre studies (12 out of the 41 included articles) could potentially offer the optimal solution (Broder, 1997; Roberts \& Shute, 2012), yet are not without their own difficulties (Sandy, Kilpatrick, Persson, Bessel, Waylen, Ness, \& Ireland, 2011).

Another central issue pertains to the lack of reference to appropriate control and comparison groups. In the present review, approximately a third of the studies had a control group, while another third compared findings with norms or other reference groups. The remaining studies did not provide any information to facilitate comparisons in findings between the patient group and the general population. This was particularly apparent within those studies exploring treatment-related experiences; only two out of eight studies included a control group.

A third challenge apparent within the CFA literature is the number of instruments used to measure the various aspects of adjustment. Even when measuring the same outcomes, a myriad of different questionnaires are used (Klassen et al., 2012), making it difficult to compare findings. Further, studies using the same measures are often built upon different designs, samples, or cultural settings, and results may be presented in different ways, so that comparisons are complicated. An additional consideration is whether to use generic measures, or employ condition-specific instruments. While generic measures of health and wellbeing allow for comparisons between CFA samples and reference groups, complementary condition-specific measures that capture the distinct domains important to particular patient groups may be needed in order to fully capture the complexities of the condition itself. Few condition-specific measures currently exist, and thus quantitative and qualitative work toward developing and evaluating such a measure may be beneficial (Klassen et al., 2012; Holmbeck \& Aspinall, 2015; Patrick, Topolski, Edwards, Aspinall, Kapp-Simon, Rumsey, Strauss, \& Thomas, 2007). 
This challenge is exacerbated by the lack of a conceptual frameworks to guide measurement (Endriga \& Kapp-Simon, 1999; Roberts \& Shute, 2012; Roberts \& Mathias, 2013). Conceptual frameworks and corresponding measures have been advocated in the related fields of oral clefts and visible difference more generally (see ARC, 2009; Klassen et al., 2012; Stock et al., 2016), and may also offer guidance for the direction of research within the field of CFAs. A clear priority for the CFA research field is to achieve consensus among researchers and clinicians regarding key outcomes and processes, followed by recommendations of useful measures and instruments, selected according to psychometric properties and their scientific and clinical meaningfulness. Such a consensus would allow researchers to address some of the key unanswered questions and would lead to a better understanding of the impact of CFAs on those affected and their families.

A fifth and more surprising finding of the current review was the overall lack of qualitative research. Qualitative studies are less common than quantitative methods in health research more generally, possibly because these approaches are not well understood and/or because lesser value is placed on them (Nelson, 2009). Nonetheless, the question of adjustment to CFAs is ideally suited to qualitative methods, due to the rare nature of the conditions, and to the current lack of knowledge about what it is like to live with a CFA (Knight et al., 2014; Holmbeck \& Aspinall, 2015).

A number of smaller points related to methodological approaches were also raised by this review. Discrepancies in the views of clinicians, parents and patients were observed within the included papers, pointing to the need to include multiple perspectives within studies. Further, interesting differences in mothers' and fathers' reports of QoL indicate that both parents' perspectives should be included, in a research field which has historically been dominated by mothers' reports (Nelson \& Kirk, 2013; Stock \& Rumsey, 2015). Future studies should also dedicate attention to the possible influence of additional physical problems, such as impaired vision or speech, and the presence of pain, as well as patients' general health perceptions.

Finally, the relatively small number of studies included in this review overall is notable. In some domains, few studies, combined with contradictory findings, complicates the accuracy of a summary and interpretation of the findings. Of further interest is that some authors appear to make assumptions between variables that were measured (e.g. satisfaction with appearancerelated treatment) and variables that were not measured (e.g. social stigma), without 
appropriate references to support these conclusions. Such observations could possibly be indicative of a lack of psychological expertise within some multidisciplinary CFA teams. The routine assessment of patients on a number of important psychological variables could yield a more holistic understanding of the patient experience (Partridge, 2010) from multiple perspectives (Roberts \& Shute, 2012), with the additional possibility of longitudinal data collection.

One methodological consideration pertaining to this review itself, is that several papers identified by the literature search included oral clefts and other CFAs within the same sample. Since several recent reviews have explored the psychological impact of oral clefts, studies related to this diagnosis were excluded, unless results were presented separately. Therefore and unfortunately, several mixed studies which could potentially have added value to the present review had to be excluded. Future research should aim to investigate potential differences and/or similarities in psychological adjustment between these two traditionally separated research fields.

\section{Conclusions}

To the authors' knowledge, this is the first literature review to comprehensively assess the state of the literature pertaining to psychological adjustment to CFAs since the overview published by Endriga and Kapp-Simon in 1999. The present review has highlighted the overall lack of literature in this area, alongside a number of important methodological challenges, which together render conclusions difficult to draw. Across the key domains of adjustment, children and adults with non-syndromic CFAs reported scores that were comparable to norms or control groups in approximately half of the included studies, while the picture appeared to be further complicated in the case of syndromic CFAs. One possible explanation for this is the association between poor adjustment and underlying developmental and/or health-related difficulties, an indication which requires further exploration. In addition, the visibility of the condition was not found to be strongly related to adjustment within the current review, in contrast to the common assumption that those with CFAs will experience significant challenges as a direct result of their visible difference.

Several methodological considerations were illustrated and discussed within the present review. In order to move the field forward, efforts should be made to strive towards larger and more homogenous samples, to include relevant control and reference groups, and to achieve consensus regarding key outcomes and measures. More consistent methodologies across 
studies would assist researchers in addressing some of the key unanswered questions and lead to a better understanding of the impact of CFAs. Given the contradictory nature of some of the present findings, much future research is needed to explore the pathways through which craniofacial differences might affect an individual's psychological adjustment, and to identify appropriate ways to prevent psychological distress and promote the development of resilience.

\section{References}

Appearance Research Collaboration. (2009). Identifying factors and processes contributing to successful adjustment to disfiguring conditions: Final report. University of the West of England: Centre for Appearance Research.

Bannink, N., Maliepaard, M., Raat, H., Joosten, K. F., \& Mathijssen, I. M. (2010). Health-related quality of life in children and adolescents with syndromic craniosynostosis. Journal of Plastic, Reconstructive, \& Aesthetic Surgery, 63, 1972-1981. doi: 10.1016/j.bjps.2010.01.036

Bannink, N., Maliepaard, M., Raat, H., Joosten, K. F., \& Mathijssen, I. M. (2011). Obstructive sleep apneaspecific quality of life and behavioral problems in children with syndromic craniosynostosis. Journal of Developmental and Behavioral Pediatrics, 32, 233-238. doi: 10.1097/DBP.0b013e318206d5e3

Beaune, L., Forrest, C. R., \& Keith, T. (2004). Adolescents' perspectives on living and growing up with Treacher Collins syndrome: a qualitative study. The Cleft Palate - Craniofacial Journal, 41, 343-350. doi: 10.1597/02-158.1

Becker, D. B., Petersen, J. D., Kane, A. A., Cradock, M. M., Pilgram, T. K., \& Marsh, J. L. (2005). Speech, cognitive, and behavioral outcomes in nonsyndromic craniosynostosis. Plastic and Reconstructive Surgery, 116, 400-407. Doi: 10.1097/01.prs.0000172763.71043.b8

Boltshauser, E., Ludwig, S., Dietrich, F., \& Landolt, M. A. (2003). Sagittal craniosynostosis: cognitive development, behaviour, and quality of life in unoperated children. Neuropediatrics, 34, 293-300. doi: $10.1055 / \mathrm{s}-2003-44667$

Broder, H. L. (1997). Psychological research of children with craniofacial anomalies: Review, critique, and implications for the future. The Cleft Palate - Craniofacial Journal, 34, 402-4.

Broder, H.L. (2001). Using psychological assessment and therapeutic strategies to enhance well-being. The Cleft Palate - Craniofacial Journal, 38, 248-54. DOI: 10.1597/1545-1569(2001)038<0248:UPAATS>2.0.CO;2

Buchanan EP, Xue AS, \& Hollier LH Jr. (2014). Craniofacial syndromes. Plastic and Reconstructive Surgery, 134, 128e-153e. doi: 10.1097/PRS.0000000000000308

Clarke, A., Thompson, A. R., Jenkinson, E., Rumsey, N., \& Newell, R. (2013). CBT for appearance anxiety: Psychosocial interventions for anxiety due to visible difference. Wiley-Blackwell

Cloonan, Y. K., Collett, B., Speltz, M. L., Anderka, M., \& Werler, M. M. (2013). Psychosocial outcomes in children with and without non-syndromic craniosynostosis: findings from two studies. The Cleft Palate Craniofacial Journal, 50, 406-413. doi: 10.1597/11-074

Cohen, M. M., Jr., Kreiborg, S., Lammer, E. J., Cordero, J. F., Mastroiacovo, P., Erickson, J. D., . . \& MartinezFrias, M. L. (1992). Birth prevalence study of the Apert syndrome. American Journal of Medical Genetics, 42, 655-659. doi: 10.1002/ajmg.1320420505

Collett, B. R., Gray, K. E., Kapp-Simon, K. A., Birgfeld, C., Cunningham, M., Rudo-Stern, J., . . \& Speltz, M. L. (2013). Laypersons' ratings of appearance in children with and without single-suture craniosynostosis. Journal of Craniofacial Surgery, 24, 1331-1335. doi: 10.1097/SCS.0b013e3182997885

de Jong, T., Maliepaard, M., Bannink, N., Raat, H., \& Mathijssen, I. M. (2012). Health-related problems and quality of life in patients with syndromic and complex craniosynostosis. Childs Nervous System, 28, 879-882. doi: 10.1007/s00381-012-1681-4

Dufton, L. M., Speltz, M. L., Kelly, J. P., Leroux, B., Collett, B. R., \& Werler, M. M. (2011). Psychosocial outcomes in children with hemifacial microsomia. Journal of Pediatric Psychology, 36, 794-805. doi: 10.1093/jpepsy/jsq112 
Endriga, M. C., \& Kapp-Simon, K. A. (1999). Psychological issues in craniofacial care: state of the art. The Cleft Palate - Craniofacial Journal, 36, 3-11. doi: 10.1597/1545-1569(1999)036<0001:piiccs>2.3.co;2

Feragen, K. B. (2012). Congenital conditions. In N. Rumsey \& D. Harcourt (Eds), The psychology of appearance (pp. 353-371). Oxford University Press.

Feragen, K. B., Stock, N. M., \& Kvalem, I. L. (2015). Risk and protective factors at age 16: Psychological adjustment in children with a cleft lip and/or palate. The Cleft Palate - Craniofacial Journal, 52, 555573. doi: 10.1597/14-063

Feragen, K.B., Stock, N.M., Sharratt, N.D., \& Kvalem, I.L. (2016). Self-perceptions of romantic appeal in adolescents with a cleft lip and/or palate. Body Image, 23, 143-152. doi: 10.1016/j.bodyim.2016.06.009

Fischer, S., Tovetjarn, R., Maltese, G., Sahlin, P. E., Tarnow, P., \& Kolby, L. (2014). Psychosocial conditions in adults with Crouzon syndrome: A follow-up study of 31 Swedish patients. Journal of Plastic Surgery and Hand Surgery, 48, 244-247. doi: 10.3109/2000656x.2013.868811

Furman, W., \& Shaffer, L. (2003). The role of romantic relationships in adolescent development. In: Adolescent romantic relations and sexual behavior: Theory, research, and practical implications, Lawrence Erlbaum Associates Publishers, Mahwah, NJ, USA (2003), pp. 3-22.

Geels, L. M., Kieffer, J. M., Hoogstraten, J., \& Prahl-Andersen, B. (2008). Oral health-related quality of life of children with craniofacial conditions. The Cleft Palate - Craniofacial Journal, 45, 461-467. doi: 10.1597/07-046.1

Geirdal, A., Overland, B., Heimdal, K., Storhaug, K., Asten, P., \& Akre, H. (2013). Association between obstructive sleep apnea and health-related quality of life in individuals affected with Treacher Collins syndrome. European Archives of Otorhinolaryngology, 270, 2879-2884. doi: 10.1007/s00405-0132409-0

Geirdal, A. O., Saltnes, S. S., Storhaug, K., Asten, P., Nordgarden, H., \& Jensen, J. L. (2015). Living with orofacial conditions: psychological distress and quality of life in adults affected with Treacher Collins syndrome, cherubism, or oligodontia/ectodermal dysplasia-a comparative study. Qualitative Life Research, 24, 927-935. doi: 10.1007/s11136-014-0826-1

Grandfield T, Thompson AR and Turpin G (2005) An attitudinal study of responses to a range of dermatological conditions using the Implicit Association Test. Journal of Health Psychology, 10, 821-829. doi: $10.1177 / 1359105305057316$

Griffiths, C., Williamson, H., \& Rumsey, N. (2012). The romantic experiences of adolescents with a visible difference: exploring concerns, protective factors and support needs. Journal of Health Psychology, 17, 1053-1064. doi: 10.1177/1359105311433909

Grogan, S. (2008). Body Image: Understanding body dissatisfaction in men, women, and children. Routledge.

Habal, M. B. (2013). Improving the quality of life of patients through pediatric plastic and craniofacial surgery. Journal of Craniofacial Surgery, 24, 21-27. doi: 10.1097/SCS.0b013e31827b3826

Holmbeck, G. N., \& Aspinall, C. L. (2015). Disorders of sex development: Lessons to be learned from studies of spina bifida and craniofacial conditions. Hormone and Metabolic Research, 47, 380-386. doi: 10.1055/s-0035-1545273

Jenkinson, E., Williamson, H., Byron-Daniel, J., \& Moss, T.P. (2015). Systematic review: Psychosocial interventions for children and young people with visible differences resulting from appearance altering conditions, injury, or treatment effects. Journal of Pediatric Psychology, 40, 1017-1033. doi: 10.1093/jpepsy/jsv048

Jonas, R. K., Montojo, C. A., \& Bearden, C. E. (2014). The 22q11.2 deletion syndrome as a window into complex neuropsychiatric disorders over the lifespan. Biological Psychiatry, 75, 351-360. doi: 10.1016/j.biopsych.2013.07.019

Kapp-Simon, K. A. (1998). Mental development and learning disorders in children with single suture craniosynostosis. The Cleft Palate - Craniofacial Journal, 35, 197-203. doi: 10.1597/15451569(1998)035<0197:mdaldi>2.3.co;2

Kapp-Simon, K. A., Collett, B. R., Barr-Schinzel, M. A., Cradock, M. M., Buono, L. A., Pietila, K. E., \& Speltz, M. L. (2012). Behavioral adjustment of toddler and preschool-aged children with single-suture craniosynostosis. Plastic and Reconstructive Surgery, 130, 635-647. doi: 10.1097/PRS.0b013e31825dc18b

Kapp-Simon, K. A., \& McGuire, D. E. (1997). Observed social interaction patterns in adolescents with and without craniofacial conditions. The Cleft Palate - Craniofacial Journal, 34, 380-384. doi: 10.1597/1545-1569(1997)034<0380:osipia>2.3.co;2 
Kapp-Simon, K. A., Speltz, M. L., Cunningham, M. L., Patel, P. K., \& Tomita, T. (2007). Neurodevelopment of children with single suture craniosynostosis: a review. Childs Nervous System, 23, 269-281. doi: 10.1007/s00381-006-0251-z

Kelleher, M. O., Murray, D. J., McGillivary, A., Kamel, M. H., Allcutt, D., \& Earley, M. J. (2006). Behavioral, developmental, and educational problems in children with nonsyndromic trigonocephaly. Journal of Neurosurgery, 105, 382-384. doi: 10.3171/ped.2006.105.5.382

Kelleher, M. O., Murray, D. J., McGillivary, A., Kamel, M. H., Allcutt, D., \& Earley, M. J. (2007). Non-syndromic trigonocephaly: Surgical decision making and long-term cosmetic results. Childs Nervous System, 23, 1285-1289. doi: 10.1007/s00381-007-0386-6

Khetani, M. A., Collett, B. R., Speltz, M. L., \& Werler, M. M. (2013). Health-related quality of life in children with hemifacial microsomia: parent and child perspectives. Journal of Development and Behavioral Pediatrics, 34, 661-668. doi: 10.1097/dbp.0000000000000006

Klassen, A.F., Tsangaris, E., Forrest, C.R., Wong, K.W., Pusic, A.L., Cano, S.J., Syed, I., Dua, M., Kainth, S., Johnson, J., \& Goodacre, T. (2012). Quality of life of children treated for cleft lip and/or palate: A systematic review. Journal of Plastic, Reconstructive \& Aesthetic Surgery, 65, 547-57. doi: 10.1016/j.bjps.2011.11.004.

Knight, S. J., Anderson, V. A., Spencer-Smith, M. M., \& Da Costa, A. C. (2014). Neurodevelopmental outcomes in infants and children with single-suture craniosynostosis: A systematic review. Developmental Neuropsychology, 39, 159-186. doi: 10.1080/87565641.2014.886690

Knudsen, E., Maltese, G., Tarnow, P., Tovetjarn, R., \& Kolby, L. (2012). Parental estimation of early psychological development in children operated on for single suture synostosis. Journal of Plastic Surgery and Hand Surgery, 46, 152-154. doi: 10.3109/2000656x.2012.676555

Kunz, M., Lehner, M., Heger, A., Armbruster, L., Weigand, H., Mast, G., \& Peraud, A. (2014). Neurodevelopmental and esthetic results in children after surgical correction of metopic suture synostosis: A single institutional experience. Childs Nervous System, 30, 1075-1082. doi: 10.1007/s00381-013-2340-0

Maliepaard, M., Mathijssen, I. M., Oosterlaan, J., \& Okkerse, J. M. (2014). Intellectual, behavioral, and emotional functioning in children with syndromic craniosynostosis. Pediatrics, 133, e1608-1615. doi: 10.1542/peds.2013-3077

McCarthy, J.G., Warren, S.M., Bernstein, J., Burnett, W., Cunningham, M.L., Edmond, J.C.... ,\& Yemen, T.A. (2012). Craniosynostosis Working Group. Parameters of care for craniosynostosis. The Cleft Palate Craniofacial Journal, 49 Suppl, 1S-24S. doi: 10.1597/11-138

Moss, T. P. (2005). The relationships between objective and subjective ratings of disfigurement severity, and psychological adjustment. Body Image, 2, 151-159. doi: 10.1016/j.bodyim.2005.03.004

Nelson, P.A. (2009). Qualitative approaches in craniofacial research. The Cleft Palate - Craniofacial Journal, 46, 245-51. doi: 10.1597/08-121.1.

Nelson, P., Glenny, A.M., Kirk, S., \& Caress, A.L. (2012). Parents' experiences of caring for a child with a cleft lip and/or palate: a review of the literature. Child: Care, Health, and Development, 38, 6-20. doi: 10.1111/j.1365-2214.2011.01244.x

Nelson, P. A., \& Kirk, S. A. (2013). Parents' perspectives of cleft lip and/or palate services: A qualitative interview. The Cleft Palate - Craniofacial Journal, 50, 275-285. doi: 10.1597/11-293

Norman, A., Moss, T.P. (2015). Psychosocial interventions for adults with visible differences: A systematic review. Peer Journal, 2,e870. doi: 10.7717/peerj.870

Okkerse, J. M., Beemer, F. A., Cordia-de Haan, M., Heineman-de Boer, J. A., Mellenbergh, G. J., \& Wolters, W. H. (2001). Facial attractiveness and facial impairment ratings in children with craniofacial malformations. The Cleft Palate - Craniofacial Journal, 38, 386-392. doi: 10.1597/15451569(2001)038<0386:faafir>2.0.co;2

Okkerse, J. M., Beemer, F. A., de Jong, T. H., Mellenbergh, G. J., Vaandrager, J. M., Vermeij-Keers, C., \& Heineman-de Boer, J. A. (2004). Condition variables in children with craniofacial anomalies: a descriptive study. Journal of Craniofacial Surgery, 15, 151-157.

Orphanet Report Series. Prevalence of rare diseases: Bibliographic data (July 2015). Retrieved from: http://www.orpha.net. Accessed March 19, 2016.

Ozgur, B. M., Aryan, H. E., Ibrahim, D., Soliman, M. A., Meltzer, H. S., Cohen, S. R., \& Levy, M. L. (2006). Emotional and psychological impact of delayed craniosynostosis repair. Childs Nervous System, 22, 1619-1623. doi: 10.1007/s00381-006-0148-x 
Partridge, J. (2010). Psycho-social reflections on craniofacial morphogenesis. Seminars in Cell Development \& Biology, 21, 333-338. doi: 10.1016/j.semcdb.2010.01.006

Partridge, J. \& Julian, D. (2008). The incidence and prevalence of disfigurement. London: Changing Faces.

Patrick, D.L., Topolski, T.D., Edwards, T.C., Aspinall, C.L., Kapp-Simon, K.A., Rumsey, N.J., Strauss, R.P., Thomas, C.R. (2007). Measuring the quality of life of youth with facial differences. The Cleft Palate-Craniofacial Journal, 44, 538-547. doi:http://dx.doi.org/10.1597/06-072.1

Philip, N., \& Bassett, A. (2011). Cognitive, behavioural and psychiatric phenotype in 22q11.2 deletion syndrome. Behavior Genetics, 41, 403-412. doi: 10.1007/s10519-011-9468-z

Pillemer, F. G., \& Cook, K. V. (1989). The psychosocial adjustment of pediatric craniofacial patients after surgery. Cleft Palate Journal, 26, 201-207.

Plomp, R. G., Mathijssen, I. M., Moolenburgh, S. E., van Montfort, K. A., van der Meulen, J. J., \& Poublon, R. M. (2015). Nasal sequelae of Treacher Collins syndrome. Journal of Plastic, Reconstructive \& Aesthetic Surgery, 68, 771-781. doi: 10.1016/j.bjps.2015.02.029

Plomp, R.G., Versnel, S.L., van Lieshout, M.J., Poublon, R.M., Mathijssen, I.M. (2013). Long-term assessment of facial features and functions needing more attention in treatment of Treacher Collins syndrome. Journal of Plastic, Reconstructive \& Aesthetic Surgery, 66, e217-26. doi: 10.1016/j.bjps.2013.03.029.

Posnick, J. C., \& Ruiz, R. L. (2000). Treacher Collins syndrome: Current evaluation, treatment, and future directions. The Cleft Palate - Craniofacial Journal, 37, 434. doi: 10.1597/15451569(2000)037<0434:tcscet>2.0.co;2

Prescott, T., Redfors, M., Rustad, C. F., Eiklid, K. L., Geirdal, A. O., Storhaug, K., \& Jensen, J. L. (2013). Characterization of a Norwegian cherubism cohort; molecular genetic findings, oral manifestations and quality of life. European Journal of Medical Genetics, 56, 131-137. doi: 10.1016/j.ejmg.2012.12.008

Raposo-Amaral, C. E., Neto, J. G., Denadai, R., Raposo-Amaral, C. M., \& Raposo-Amaral, C. A. (2014). Patientreported quality of life in highest-functioning Apert and Crouzon syndromes: a comparative study. Plastic and Reconstructive Surgery, 133, 182e-191e. doi: 10.1097/01.prs.0000437260.31693.75

Raposo-Amaral, C. E., Raposo-Amaral, C. A., Garcia Neto, J. J., Farias, D. B., \& Somensi, R. S. (2012). Apert syndrome: quality of life and challenges of a management protocol in Brazil. Journal of Craniofacial Surgery, 23, 1104-1108. doi: 10.1097/SCS.0b013e318258814a

Roberts, R. M. (2014). Discrimination among adults with craniofacial conditions. Journal of Craniofacial Surgery, 25, 77-81. doi: 10.1097/SCS.0b013e3182a2e005

Roberts, R. M., \& Mathias, J. L. (2012). Psychosocial functioning in adults with congenital craniofacial conditions. The Cleft Palate - Craniofacial Journal, 49, 276-285. doi: 10.1597/10-143

Roberts, R. M., \& Mathias, J. L. (2013). Predictors of mental health in adults with congenital craniofacial conditions attending the Australian craniofacial unit. The Cleft Palate - Craniofacial Journal, 50, 414423. doi: $10.1597 / 11-105$

Roberts, R. M., \& Shute, R. H. (2012). A prospective study of coping and adjustment in adolescents with craniofacial conditions. Children's Health Care, 41, 111-128. Doi: 10.10809/02739615.2012.657031.

Rosenberg, J. M., Kapp-Simon, K. A., Starr, J. R., Cradock, M. M., \& Speltz, M. L. (2011). Mothers' and fathers' reports of stress in families of infants with and without single-suture craniosynostosis. The Cleft Palate - Craniofacial Journal, 48, 509-518. doi: 10.1597/09-210

Rumsey, N., \& Harcourt, D. (2004). Body image and disfigurement: issues and interventions. Body Image, 1, 8397. doi: 10.1016/s1740-1445(03)00005-6

Sandy, J., Kilpatrick, N., Persson, M., Bessel, A., Waylen, A., Ness, A., \& Ireland, A. (2011). Why are multi-centre clinical observational studies still so difficult to run? British Dental Journal, 211, 59-61. doi: 10.1038/sj.bdj.2011.570

Sarimski, K. (2001). Social adjustment of children with a severe craniofacial anomaly (Apert syndrome). Child: Care, Health and Development, 27, 583-590.

Sarwer, D. B., \& Crerand, C. E. (2002). Psychological issues in patient outcomes. Facial Plastic Surgery, 18, 125133. doi: $10.1055 / \mathrm{s}-2002-32203$

Seaton, C. L. (2009). Psychological adjustment. In S. J. Lopez (Ed), The encyclopedia of positive psychology. Blackwell: reference online. doi: 10.1111/b.9781405161251.2009.x

Snyder, H., \& Pope, A. W. (2010). Psychosocial adjustment in children and adolescents with a craniofacial anomaly: Diagnosis-specific patterns. The Cleft Palate - Craniofacial Journal, 47, 264-272. doi: $10.5555 / 08-227.1$ 
Speltz, M. L., Kapp-Simon, K. A., Cunningham, M., Marsh, J., \& Dawson, G. (2004). Single-suture craniosynostosis: a review of neurobehavioral research and theory. Journal of Pediatric Psychology, 29, 651-668. doi: 10.1093/jpepsy/jsh068

Speltz, M. L., Morton, K., Goodell, E. W., \& Clarren, S. K. (1993). Psychological functioning of children with craniofacial anomalies and their mothers: Follow-up from late infancy to school entry. The Cleft Palate - Craniofacial Journal, 30, 482-489. doi: 10.1597/1545-1569(1993)030<0482:pfocwc>2.3.c0;2

Stavropoulos, D., Hallberg, U., Mohlin, B., \& Hagberg, C. (2011). Living with Crouzon syndrome: How do young adults with Crouzon syndrome handle their life situation? International Journal of Paediatric Dentistry, 21, 35-42. doi: 10.1111/j.1365-263X.2010.01079.x

Stock, N. M., \& Feragen, K. B. (2016). Psychological adjustment to cleft lip and/or palate: A narrative review of the literature. Psychology and Health, 22, 1-72. doi: 10.1080/08870446.2016.1143944

Stock, N. M., Hammond, V., Owen, T., Kiff, J., Shanly, A., \& Rumsey, N. (2016). Achieving consensus in the measurement of psychological adjustment to cleft lip and/or palate. The Cleft Palate -Craniofacial Journal, 53, 421-426. doi: 10.1597/15-071

Stock NM, Rumsey N (2015). Parenting a child with a cleft: The father's perspective. The Cleft PalateCraniofacial Journal, 51: 31-43. doi.org/10.1597/13-035

Stock NM, Whale K, Jenkinson E, Rumsey N, Fox F (2013). Young people's perceptions of visible difference. Diversity and Equality in Health and Care, 10, 41-51.

Swillen, A., \& McDonald-McGinn, D. (2015). Developmental trajectories in 22q11.2 deletion. American Journal of Medical Genetics Part C Seminars of Medical Genetics, 169, 172-181. doi: 10.1002/ajmg.c.31435

Tovetjarn, R., Tarnow, P., Maltese, G., Fischer, S., Sahlin, P. E., \& Kolby, L. (2012). Children with Apert syndrome as adults: A follow-up study of 28 Scandinavian patients. Plastic and Reconstructive Surgery, 130, 572e-576e. doi: 10.1097/PRS.0b013e318262f355

van der Vlugt, J. J., van der Meulen, J. J., Creemers, H. E., Verhulst, F. C., Hovius, S. E., \& Okkerse, J. M. (2012). Cognitive and behavioral functioning in 82 patients with trigonocephaly. Plastic and Reconstructive Surgery, 130, 885-893. doi: 10.1097/PRS.0b013e318262f21f

van der Vlugt, J. J., van der Meulen, J. J., Creemers, H. E., Willemse, S. P., Lequin, M. L., \& Okkerse, J. M. (2009). The risk of psychopathology in children with craniosynostosis. Plastic and Reconstructive Surgery, 124, 2054-2060. doi: 10.1097/PRS.0b013e3181bcf2dc

Warschausky, S., Kay, J. B., Buchman, S., Halberg, A., \& Berger, M. (2002). Health-related quality of life in children with craniofacial anomalies. Plastic and Reconstructive Surgery, 110, 409-414.

Werler, M. M., Starr, J. R., Cloonan, Y. K., \& Speltz, M. L. (2009). Hemifacial microsomia: From gestation to childhood. Journal of Craniofacial Surgery, 20, 664-669. doi: 10.1097/SCS.0b013e318193d5d5

Wong-Gibbons, D. L., Kancherla, V., Romitti, P. A., Tyler, M. C., Damiano, P. C., Druschel, C. M., ... Burnett, W. (2009). Maternal reports of satisfaction with care and outcomes for children with craniosynostosis. Journal of Craniofacial Surgery, 20, 138-142. doi: 10.1097/SCS.0b013e318190e28c 